IASSNS-HEP-93/65

NSF-ITP-93-128

OSU-M-93-2

UTTG-24-93

\title{
Mirror Symmetry for Hypersurfaces in Weighted Projective Space and Topological Couplings
}

\author{
Per Berglund \\ School of Natural Science \\ Institute for Advanced Study \\ Olden Lane \\ Princeton, NJ 08540 \\ berglund @ guinness.ias.edu \\ and \\ Sheldon Katz \\ Department of Mathematics \\ Oklahoma State University \\ Stillwater, OK 74078 \\ katz@math.okstate.edu
}

\begin{abstract}
By means of toric geometry we study hypersurfaces in weighted projective space of dimension four. In particular we compute for a given manifold its intrinsic topological coupling. We find that the result agrees with the calculation of the corresponding coupling on the mirror model in the large complex structure limit.
\end{abstract}

December 1993 


\section{Introduction}

Mirror symmetry [1] is in one respect the result of a very simple observation; at the level of the $(2,2)$ superconformal field theory the relative sign of the $U(1)$-current is ambiguous and the two different choices lead to isomorphic theories [2]. On the other hand, when the theory is formulated as a two-dimensional $N=2$ supersymmetric non-linear $\sigma$-model on a Calabi-Yau manifold as the target space the result is far from trivial. The change of sign of the $U(1)$-current interchanges the $(c, c)$ and the $(a, c)$ ring and so the effect is to flip the sign of the Euler number, $\chi_{E}=2\left(b_{1,1}-b_{2,1}\right)$, thus relating two topologically distinct manifolds. This has far-reaching consequences. In particular by computing the $(2,1)$ form couplings on $\mathcal{W}$, the mirror of $\mathcal{M}$, we obtain, by mirror symmetry, the fully corrected $(1,1)$ form couplings on $\mathcal{M}$. This statement is true for all of moduli space and hence we know the 'quantum' Kähler couplings not only in the large radius limit but at any point in the space of Kähler deformations of $\mathcal{M}$.

Based on the recent classification efforts of $N=2$ string vacua [3, 4, 5] the class of known $(2,2)$ Landau-Ginzburg orbifolds is (almost) mirror symmetric. Still mirror symmetry is merely a conjecture and the number of models for which mirror symmetry has been explicitly checked is rather small [6, 7, 8, 9] . In this paper we continue the effort of verifying mirror symmetry. Following Candelas et al. [6] we study a one-dimensional subspace of the space of complex deformations of a class of manifolds $\mathcal{W}$ whose mirrors are transverse hypersurfaces $\mathcal{M}$ in weighted $\mathbb{P}_{\left(k_{1}, \ldots, k_{5}\right)}^{4}$. We restrict to the deformation which corresponds to the Kähler form inherited from the ambient space of $\mathcal{M} \mathbb{1}$ It has been argued that such a deformation always exists for a generic choice of the defining polynomial for $\mathcal{W}$ [10], and we will show that this is indeed the case, using the construction of mirror manifolds proposed by Batyrev [11]. With this information at hand we find the periods which are solutions of the Picard-Fuchs equation [12]. The existence of this equation follows from the fact that the moduli space is not just Kähler but 'special Kähler' due to the $N=2$ world-sheet supersymmetry [13, 14]. The knowledge of the periods allows us to solve the theory completely. In particular, we calculate the Yukawa coupling as a function on our one parameter subspace of the moduli space. In the large complex structure limit this will give predictions for the topological Kähler coupling $y_{J J J}$ on $\mathcal{M}$. Indeed, this is confirmed by an intersection calculation where we find that the couplings are exactly the same (see (2.4) and (5.14)). This latter unexpected agreement suggests that it may be possible to formulate the computation only in terms of the Kähler modulus dual to the fundamental monomial. In fact, once the fundamental period has been found we may think of the computation as carried out on the Kähler moduli space of $\mathcal{M}$ rather than on the space of complex deformations of $\mathcal{W}$. Special geometry is a property not just of the complex deformations but for the Kähler deformations as well. Hence, one might expect that when formulating the theory completely on the Kähler side the same Picard-Fuchs

1 In general, due to the quotient singularities from the projectivization, it is $k$ times the naïve Kähler form which is well-defined. The weight $k$ of the hyperplane class of $\mathcal{M}$ is a natural number which depends on the singularity, see section 2 and appendix A. 
equation will appear. This would not only allow us to solve the theory completely within $\mathcal{M}$ but it would also give a proof of mirror symmetry for all of moduli space. See also [15].

The paper is organized as follows. In section 2 we derive an expression for the topological Yukawa coupling $y_{J J}$ on $\mathcal{M}$. We then discuss some basic properties of toric geometry needed to compute the period (section 3) as well as to understand the complex structure moduli space of the $\mathcal{W}$ (section 4 ). In section 5 the Yukawa coupling $y_{\psi \psi \psi}^{\text {lcs }}$ in the large complex structure limit on $\mathcal{W}$ is obtained from the knowledge of the monodromy around $\psi=\infty$. Comparison with $y_{J J J}$ on $\mathcal{M}$ gives complete agreement for all models studied so far. Our conclusions and discussions are left for section 6 while some technical details regarding the analytic continuation of the periods and the derivation of the weight $k$ of the hyperplane class of $\mathcal{M}$ can be found in the appendices.

\section{The Topological Yukawa Coupling}

Let $p\left(x_{i}\right)=0$ define a hypersurface $\mathcal{M} \in \mathbb{P}_{\left(k_{1}, k_{2}, k_{3}, k_{4}, k_{5}\right)}^{4}[d]$ where $p\left(x_{i}\right)$ is a quasi homogeneous polynomial of degree $d=\sum_{i} k_{i}$. 2 Recall that the $x_{i}$ are weighted coordinates,

$$
x_{i} \sim \lambda^{k_{i}} x_{i} i=1, \ldots, 5 ; \quad p\left(\lambda^{k_{i}} x_{i}\right) \sim \lambda^{d} p\left(x_{i}\right) .
$$

Associated to $\mathcal{M}$ is the parameter space of Kähler deformations and complex structure deformations of dimension $h_{1,1}=\operatorname{dim} H^{1,1}(\mathcal{M})$ and $h_{2,1}=\operatorname{dim} H^{2,1}(\mathcal{M})$ respectively3. We are interested in computing the $(1,1)$ form Yukawa coupling restricted to the case when all three $(1,1)$ forms are equal to the Kähler form $J \in H^{1,1}(\mathcal{M})$ inherited from the ambient space,

$$
y_{J J J}=\int_{\mathcal{M}} J^{3}=H^{3},
$$

where $H \in H^{2}(\mathcal{M})$ is the hyperplane class and $H^{3}$ is the triple intersection number of $H$. This coupling is topological, i.e. it does not depend on the choice of complex structure of $\mathcal{M}$; neither do any of the other $(1,1)$-couplings. In the light of the recent results on topology change in string theory [16] one may worry that the intersection number will depend on which large radius limit is chosen. However, the topology change arises from choosing different desingularizations of $\mathcal{M}$, and since $H^{3}$ can be calculated on any of these by pulling back the calculation for $\mathcal{M}$, the intersection number is well defined. In contrast to the $(2,1)$ form coupling, $y_{J J J}$ will receive quantum corrections in the form of instanton contributions [17]. However, for large radius this contribution is exponentially suppressed and $y_{J J}$ will give the correct result of the corresponding three-point function

2 As usual $\mathbb{P}_{\left(k_{1}, k_{2}, k_{3}, k_{4}, k_{5}\right)}^{4}[d]$ refers to the family of degree $d$ hypersurfaces in the weighted projective space $\mathbb{P}_{\left(k_{1}, k_{2}, k_{3}, k_{4}, k_{5}\right)}^{4}$.

3 We actually mean the dimensions of the spaces of harmonic $(1,1)$ and $(2,1)$ forms on an appropriate Calabi-Yau desingularization of $\mathcal{M}$. 
in the underlying conformal field theory. Thus, in this limit, we have the possibility of comparing calculations made on $\mathcal{M}$ and $\mathcal{W}$.

For those models in which the projectivity constraint (2.1) does not lead to any singularities on the manifold, hence no extra $(1,1)$ forms,

$$
y_{J J J}=\frac{d}{\prod_{i=1}^{5} k_{i}} .
$$

However, eq. (2.3) holds only for four hypersurfaces in weighted $\mathbb{P}^{4}$. Rather, there are singularities on the manifold which when blown up contribute to the number of $(1,1)$ forms and also change (2.3). As is shown in appendix A, the effect on $y_{J J}$ leads to a very simple correction of (2.3),

$$
y_{J J}=\frac{d k^{3}}{\prod_{i=1}^{5} k_{i}},
$$

where $k$, the weight of the hyperplane class of $\mathcal{M}$, is a natural number. In appendix A we give a derivation of $k$ for any hypersurface in a weighted projective space of dimension four. Below we will, following [8], compute $y_{J J J}$ explicitly for two examples as well as use (2.4) for comparison. Related intersection numbers have been computed in [9].

Example 1. Consider the hypersurface $\mathcal{M}_{1} \in \mathbb{P}_{(1,1,1,2,2)}^{4}[7]$ defined by $p_{1}=0$ where $p_{1}$ is a transverse polynomial. Note that $p_{1}$ cannot be a Fermat. There is a $\mathbb{Z}_{2}$ fixed point set given by a $\mathbb{P}^{1}$. From (A.3) we have $k=2$ for this example. Let $H$ be the linear system of quadratic polynomials. We are interested in computing the triple intersection $H^{3}$. Since $x_{4}$ and $x_{5}$ have degree 2 , both $x_{4}=$ const and $x_{5}=$ const are in $H$. Thus, in the patch $x_{1}=1$ we can describe $H^{3}$ by the intersection of $x_{4}=$ const, $x_{5}=$ const and a quadratic equation in $x_{2}$ and $x_{3}$. If we choose $p_{1}=x_{1}^{7}+x_{2}^{7}+x_{2} x_{4}^{3}+x_{3}^{7}+x_{3} x_{5}^{3}$ we get $x_{2}^{7}+x_{3}^{7}=$ const which together with the quadratic equation in $x_{2}$ and $x_{3}$ form a system of equations with $2 \cdot 7=14$ solutions. Thus,

$$
H^{3}=14
$$

agreeing with (2.4).

Example 2. As the second example we will consider a model $\mathcal{M}_{2}$ for which we do not know how to construct the mirror manifold $\mathcal{W}_{2}$ by means of the transposition argument [18]. Let $\mathcal{M}_{2} \in \mathbb{P}_{(1,1,3,4,6)}^{4}[15]$ be defined by a transverse polynomial $p_{2}=0$. The projectivity condition (2.1) gives a $\mathbb{Z}_{12}$ fixed point set and from (A.3) $k=12$. $H$ is given by polynomials of order 12 , e.g. $x_{5}^{2}$. So by setting $x_{5}^{2}=$ const, $x_{4}^{3}=$ const and $x_{3}^{4}=$ const they describe together with $p_{2}=x_{1}^{15}+x_{2}^{15}+x_{3}^{5}+x_{3} x_{4}^{3}+x_{3} x_{5}^{2}+x_{2} x_{4}^{2} x_{5}$ the intersection $H^{3}$. This system has $2 \cdot 3 \cdot 4 \cdot 15=360$ solutions. Thus,

$$
H^{3}=360,
$$

in agreement with (2.4).

In section 5 we return to mirror models of the respective examples where we compute the corresponding (2,1)-form coupling in the large complex structure limit. By mirror symmetry the two types of couplings are supposed to agree. As will be shown this is indeed the case hence verifying mirror symmetry (at one point in moduli space) for a large class of models. 


\section{The Fundamental Period}

Recall that the structure of the moduli space of the complex structure is determined by the period vector $\varpi_{i}[19,20,21]$. Let $\hat{p}_{\phi_{j}}\left(y_{i}\right)=0$ be a generic hypersurface in the family $\mathbb{P}_{\left(k_{1}, k_{2}, k_{3}, k_{4}, k_{5}\right)}^{4}[d]$. Here $\phi_{j}$ denote certain local coordinates on the moduli space. The periods are integrals of the holomorphic three form $\Omega\left(\phi_{j}\right)$ over a basis of three cycles $\gamma_{i}$, $\varpi_{i}=\int_{\gamma_{i}} \Omega\left(\phi_{j}\right)$. The fundamental period of $\Omega\left(\phi_{j}\right)$ is then given by

$$
\varpi_{0}\left(\phi_{j}\right) \stackrel{\text { def }}{=} \oint_{B_{0}} \Omega\left(\phi_{j}\right)=\frac{-\psi d}{(2 \pi i)^{5}} \oint_{b_{0}} \frac{\prod_{i=1}^{5} \mathrm{~d} y_{i}}{\hat{p}_{\phi_{j}}\left(y_{i}\right)} .
$$

where $\psi=\phi_{0}$ is up to a constant the coefficient of the term $\prod_{i=1}^{5} y_{i}$ in $\hat{p}_{\phi_{j}}$. The integral has been pulled back to the affine space $\mathbb{C}_{\left(k_{1}, k_{2}, k_{3}, k_{4}, k_{5}\right)}^{5}$ and $b_{0}=\left\{y_{i}|| y_{i} \mid=\delta, i=1, \ldots, 5\right\}$ is determined so as to reproduce the integral of $\Omega\left(\phi_{j}\right)$ over the fundamental cycle $B_{0}$.

In this section we will derive an expression for the fundamental period associated to a particular one-dimensional deformation of the mirror manifold $\hat{\mathcal{W}}$ of a partial desingularization $\hat{\mathcal{M}}$ of a hypersurface $\mathcal{M} \in \mathbb{P}_{\left(k_{1}, k_{2}, k_{3}, k_{4}, k_{5}\right)}^{4}[d]$. This deformation consists of a sum of five monomials plus a multiple of a monomial which may be thought of as $\prod_{i=1}^{5} y_{i}$. The derivation will be made twice. First, the calculation will be carried out for a class of models in which $\mathcal{M}$ is defined by the zero locus of a polynomial $p$, where it is assumed that $p$ is 'invertible' [3]. By 'invertible' we mean that there exists a polynomial $p_{0}$ consisting of five terms such that $p_{0}$ is transverse, i.e. $\mathrm{d} p_{0}=p_{0}=0$ has the origin as its only solution. Then the fundamental deformation of $\mathcal{W}$ is given by adding on multiples of the monomial $\prod_{i=1}^{5} y_{i}$, where the $y_{i}$ are projective coordinates on $\mathcal{W}$. After this explicit calculation, the calculation will be redone in greater generality via toric geometry and Batyrev's proposed construction of mirror manifolds. In this way, we will be able to relate the two approaches when they overlap and hence give more evidence for why the construction discussed in [18] gives the mirror partner.

Let $\mathcal{M}$ be as in section 2, i.e. a hypersurface in $\mathbb{P}_{\left(k_{1}, k_{2}, k_{3}, k_{4}, k_{5}\right)}^{4}$ defined by $p\left(x_{i}\right)=0$ but with $p\left(x_{i}\right)$ an invertible (quasi)homogeneous polynomial of degree $d$. The mirror of $\mathcal{M}$ is an orbifold, $\mathcal{W}=\tilde{\mathcal{W}} / H$ where $\tilde{\mathcal{W}}$ is a hypersurface of degree $\hat{d}$ defined by $\hat{p}\left(y_{i}\right)=0$ in $\mathbb{P}_{\left(\hat{k}_{1}, \hat{k}_{2}, \hat{k}_{3}, \hat{k}_{4}, \hat{k}_{5}\right)}^{4}$ obtained by transposing $p$ [18].

We want to calculate the fundamental period for the one-dimensional subspace of the complex structure moduli space of $\mathcal{W}$ corresponding to deformations along the direction of $\prod_{i=1}^{5} y_{i}$, i.e. deformations of $\hat{p}_{0}$ given by

$$
\hat{p}_{\psi}=\hat{p}_{0}-\psi d y_{1} y_{2} y_{3} y_{4} y_{5}
$$

There are several ways of obtaining an explicit expression for $\varpi_{0}$. On the one hand, following the construction by Candelas et al. [6], one can perform the integration explicitly. This is the lead we will follow. On the other hand (3.1) satisfies the so-called Picard-Fuchs equation [22]. In the context of string compactifications, the existence of this differential 
equation is a general result that follows from the fact that the models we are considering are $(2,2)$-superconformal string vacua [23,24,25]. The Picard-Fuchs equation can be derived from (3.1) by using Griffiths 'reduction of pole order' analysis [12]. (For examples, see [24, [7, 23,9].) However, for explicit calculations it is preferable to use the first method, especially when studying multi-dimensional moduli spaces, (See also [9].)

Leaving the general discussion behind we now go on to compute the integral. Following [26] we expand the denominator around large $\psi$ and evaluate the integral by residues. Leaving the combinatorics to the reader we straightforwardly obtain the fundamental period as

$$
\varpi_{0}=\sum_{m=0}^{\infty} \frac{\Gamma(d m+1)}{\prod_{i=1}^{5} \Gamma\left(k_{i} m+1\right)(d \psi)^{d m}} .
$$

The fundamental period can also be written in terms of a generalized hypergeometric function,

$$
{ }_{d-1} F_{d-2}(\overbrace{\frac{1}{d}, \ldots, \frac{d-1}{d}} ; \overbrace{\frac{1}{k_{1}}, \ldots, \frac{k_{1}-1}{k_{1}}, \ldots, \frac{1}{k_{5}}, \ldots, \frac{k_{5}-1}{k_{5}}}, 1,1,1 ;\left(\prod_{i=1}^{5} k_{i}^{k_{i}} \psi\right)^{-d})
$$

This form will turn out to be useful when studying the mirror map in the large complex structure limit. The overbraces indicate that indices which are common are to be left out. In general $\varpi_{0}$ is a ${ }_{q} F_{q-1}$ where $q \leq(d-1)$. In fact, it is believed that $q$ is the number of periods which are related to the polynomial deformations.

Before generalizing the above via toric geometry, we review Batyrev's proposed toric construction of mirror pairs [11], as enhanced by the discussion of the monomial-divisor mirror map of Aspinwall-Greene-Morrison [27]. The discussion will also serve as a basis for understanding the moduli space as a toric variety. This is useful for the computation of the Yukawa coupling in the large complex structure limit.

Consider an $n$ dimensional integral polyhedron $P$, whose vertices lie in an $n$ dimensional lattice $M$ in $M_{\mathbb{R}}:=M \otimes \mathbb{R}$. One associates to $P$ a toric variety $\mathbb{P}(P)$ [28], whose dimension is the same as that of $M$. The construction also gives a canonical embedding $\mathbb{P}(P) \hookrightarrow \mathbb{P}^{|P|-1}$, where $|P|$ is the cardinality of $P \cap M$, and the coordinates of $\mathbb{P}^{|P|-1}$ are identified with the points of $P \cap M$. Consider the algebraic torus $T=\operatorname{Hom}\left(\mathrm{M}, \mathbb{C}^{*}\right) \simeq\left(\mathbb{C}^{*}\right)^{\mathrm{n}}$, and identify $M$ with the lattice of characters $\operatorname{Hom}\left(\mathrm{T}, \mathbb{C}^{*}\right)$ of $T$. $T$ may be embedded in $\mathbb{P}^{|P|-1}$ via the map defined by $t \mapsto\left(m_{1}(t), \ldots, m_{|P|}(t)\right)$, where the $m_{i}$ range over the points of $P \cap M$, identified as characters (sometimes called monomials) of $T$. The toric variety $\mathbb{P}(P)$ is in fact the closure of this map. We will often think of $\mathbb{P}(P)$ as a projective variety in this fashion without further comment.

A reflexive polyhedron is an integral polyhedron containing 0 in its interior, such that each facet of $P$ (that is, a codimension 1 face of $P$ ) is supported by a hyperplane $H$ which

4 For $(2,2)$ Landau-Ginzburg models with $n>5$ superfields it is not always clear how to perform the generalization of the integral in (3.1) . 
can be defined by a linear equation of the form $H=\left\{y \in M_{\mathbb{R}} \mid\langle\ell, y\rangle=-1\right\}$ for some $\ell$ in $N:=\operatorname{Hom}(\mathrm{M}, \mathbb{Z})$. Note that $N$ and $M$ are dual lattices, and $N$ is canonically identified with the lattice $\operatorname{Hom}\left(\mathbb{C}^{*}, \mathrm{~T}\right)$ of one parameter subgroups of $T$. Batyrev shows that if $P$ is reflexive, then the general hyperplane section of $\mathbb{P}(P)$ is Calabi-Yau (possibly with mild singularities, which can be resolved to obtain a Calabi-Yau manifold). Put $N_{\mathbb{R}}:=N \otimes \mathbb{R}$. The polar polyhedron (which Batyrev calls the dual polyhedron) is given by

$$
P^{\circ}=\left\{x \in N_{\mathbb{R}} \mid\langle x, y\rangle \geq-1 \text { for all } y \in P\right\},
$$

and is reflexive if and only if $P$ is reflexive. Batyrev proposes that the hyperplane sections $\overline{\mathcal{M}}$ of $\mathbb{P}(P)$ and $\overline{\mathcal{W}}$ of $\mathbb{P}\left(P^{\circ}\right)$ should form a mirror pair.

Consider the weighted projective space $\mathbb{P}_{\left(k_{1}, k_{2}, k_{3}, k_{4}, k_{5}\right)}^{4}$, and let $d=\sum_{i} k_{i}$. The study of hypersurfaces of degree $d$ in $\mathbb{P}_{\left(k_{1}, k_{2}, k_{3}, k_{4}, k_{5}\right)}^{4}$ is related to the study of hyperplane sections of the variety obtained as the image of the mapping $f$ from $\mathbb{P}_{\left(k_{1}, k_{2}, k_{3}, k_{4}, k_{5}\right)}^{4}$ to $\mathbb{P}^{|P|-1}$ defined by $f(x)=\left(m_{i}(x)\right)$, as $m_{i}$ ranges over all $(|P|)$ degree $d$ monomials in $\mathbb{P}_{\left(k_{1}, k_{2}, k_{3}, k_{4}, k_{5}\right)}^{4}$. The mapping $f$ need not be defined everywhere, so that it is only a rational mapping. Let $V$ be the closure of the image of $f$. But from the above discussion, we have a nice description of $V$. It is natural to define $P$ as the convex hull of the set of exponents of all degree $d$ monomials in $x_{1}, \ldots, x_{5}$. To make contact with the definition of reflexivity given above, we will need to translate $P$ by the vector $(-1,-1,-1,-1,-1)$. The translate is needed to make the origin a point of $P$. So let

$$
M=\left\{x \in \mathbb{Z}^{5} \mid \sum k_{i} x_{i}=0\right\}
$$

where the restriction is equivalent to the Calabi-Yau condition $\sum_{i=1}^{5} k_{i}=d$. In terms of $M$, which is a rank 4 lattice, we define

$$
P=\text { the convex hull of }\left\{x \in M \mid x_{i} \geq-1 \forall i\right\}
$$

It is then clear that $V=\mathbb{P}(P)$. When the weights $k_{i}$ need emphasis, we will write $P_{\vec{k}}$ in place of $P$, where $\vec{k}$ is short for $\left(k_{1}, \ldots, k_{5}\right)$.

Since we want our hyperplane sections to have Calabi-Yau resolutions, we must now make the assumption on $\left(k_{1}, \ldots, k_{5}\right)$ that $P_{\vec{k}}$ is reflexive. In particular, $\operatorname{dim} P_{\vec{k}}=4$, which leads quickly to the conclusion that $V$ is birational to $\mathbb{P}_{\left(k_{1}, k_{2}, k_{3}, k_{4}, k_{5}\right)}^{4}$. The classification of reflexive polyhedra in dimension 4 has not been done. However, there is a result which shows that there are many examples of $\vec{k}$ for which $P_{\vec{k}}$ is reflexive. Before stating it, recall the map $\rho: \mathbb{P}^{4} \rightarrow \mathbb{P}_{\left(k_{1}, k_{2}, k_{3}, k_{4}, k_{5}\right)}^{4}$ defined by $\rho\left(x_{1}, \ldots, x_{5}\right)=\left(x_{1}^{k_{1}}, \ldots, x_{5}^{k_{5}}\right)$. Let $\alpha_{j}=$ $e^{2 \pi i / k_{j}}$. Then $\rho$ exhibits $\mathbb{P}_{\left(k_{1}, k_{2}, k_{3}, k_{4}, k_{5}\right)}^{4}$ as an orbifold of $\mathbb{P}^{4}$ by the group $\mathbb{Z}_{k_{1}} \times \cdots \times \mathbb{Z}_{k_{5}}$, since $\rho$ respects the automorphism $\left(x_{1}, \ldots, x_{5}\right) \mapsto\left(\alpha^{r_{1}} x_{1}, \ldots, \alpha^{r_{5}} x_{5}\right)$ of $\mathbb{P}^{4}$ for any integers $r_{i}$.

Let $\mathcal{M} \subset \mathbb{P}_{\left(k_{1}, k_{2}, k_{3}, k_{4}, k_{5}\right)}^{4}$ be a degree $d$ hypersurface, and let $X=\rho^{-1}(\mathcal{M}) \subset \mathbb{P}^{4}$. We say that $\mathcal{M}$ is quasismooth if $X$ is smooth. Then the following is shown in [29]. 
Proposition. Suppose there is a quasismooth degree $d$ hypersurface $\mathcal{M} \subset \mathbb{P}_{\left(k_{1}, k_{2}, k_{3}, k_{4}, k_{5}\right)}^{4}$. Then $P_{\vec{k}}$ is reflexive.

These weighted projective spaces have been classified [4]. There are 7555 of them. In addition, there are many other examples of $\mathbb{P}_{\left(k_{1}, k_{2}, k_{3}, k_{4}, k_{5}\right)}^{4}$ for which $P=P_{\vec{k}}$ is reflexive [29].

There are 5 coordinate functions on the $\mathbb{Z}^{5}$ which contains $M$ according to (3.6); restricting to $M$, these are naturally thought of as elements $v_{1}, \ldots, v_{5}$ of $N$, where $v_{i}(x)=$ $x_{i}$ for $x \in M$. From the definitions (3.5) and (3.7), it follows immediately that $P^{\circ}$ contains the six points

$$
\overrightarrow{0}, v_{1}, v_{2}, v_{3}, v_{4}, v_{5}
$$

To these six points are associated respective linear coordinate functions $m_{i}$ on $\mathbb{P}\left(P^{\circ}\right)$, with $0 \leq i \leq 5$, coming from the projective embedding of $\mathbb{P}\left(P^{\circ}\right)$ and our earlier discussion.

The $v_{i}$ are linearly dependent; the only relation between them (up to scalar) is $\sum_{i} k_{i} v_{i}=0$. This leads to the relation

$$
\prod_{i=1}^{5} m_{i}^{k_{i}}=m_{0}^{d}
$$

between the $m_{i}$. It is easy to see that all relations between the $m_{i}$ are just powers of this one.

Choose the one parameter family of hypersurfaces

$$
\sum_{i=1}^{5} m_{i}-\psi d m_{0}=0
$$

In [30], a natural period is calculated for toric hypersurfaces; when this result is applied to the family (3.9), the fundamental period (3.3) is once again obtained. Since these two techniques are rather different, it is reassuring that the results agree.

In fact (3.9) and the family it describes can be related to the one parameter family (3.2) in $\mathbb{P}_{\left(\hat{k}_{1}, \hat{k}_{2}, \hat{k}_{3}, \hat{k}_{4}, \hat{k}_{5}\right)}^{4}[\hat{d}]$ by a fractional transformation. However, there are identifications due to the non-linear change of variables. These are precisely described by performing a quotient by $H$. Thus, in those cases when the transposition scheme can be performed the result is recovered by the more general toric construction of Batyrev, and the families (3.2) and (3.9) are identified [29,31]. Not surprisingly, it can be shown that the integration cycle used in [30] coincides with the integration cycle considered in (3.1) after the identifications are made.

Example: Consider a one parameter family of deformations $\mathcal{W}=\tilde{\mathcal{W}} / G$ where $\tilde{\mathcal{W}} \in$ $\mathbb{P}_{(1,3,3,3,5)}^{4}[15]$ is defined by

$$
\hat{p}_{\psi}=\hat{p}_{0}-10 \psi \prod_{i=1}^{5} y_{i}=y_{1}^{10} y_{5}+y_{5}^{3}+y_{2}^{5}+y_{3}^{5}+y_{4}^{5}-10 \psi \prod_{i=1}^{5} y_{i},
$$


and $G=\left(\mathbb{Z}_{5}: 4,1,0,0,0\right) \times\left(\mathbb{Z}_{5}: 4,0,1,0,0\right) .5$ The peculiar form of $\hat{p}_{0}$ is due to it being the transpose of $p_{0}=x_{1}^{10}+x_{1} x_{5}^{3}+x_{2}^{5}+x_{3}^{5}+x_{4}^{5}$, where $p_{0}=0$ is the mirror $\mathcal{M} \in \mathbb{P}_{(1,2,2,2,3)}^{4}[10]$ of $\mathcal{W}$. We want to relate (3.10) to the corresponding realization of $\mathcal{W}$ as a hypersurface in a toric variety given by $(3.9)$. To do so we make the identifications

$$
m_{0}=y_{1} y_{2} y_{3} y_{4} y_{5}, \quad m_{1}=y_{1}^{10} y_{5}, \quad m_{i}=y_{i}^{5}, i=2,3,4, \quad m_{5}=y_{5}^{3},
$$

where $m_{1} m_{2}^{2} m_{3}^{2} m_{4}^{2} m_{5}^{3}=m_{0}^{10}$ from (3.8). This map is not well defined since the $m_{i}$ are invariant under a $\mathbb{Z}_{3} \times \mathbb{Z}_{5}^{3}$ action generated by $G \times\left(\mathbb{Z}_{15}: 1,3,3,3,5\right)$ on the $y_{i} ; m_{0}$ transforms under a $\mathbb{Z}_{10}$ under the group of rescalings of the $y_{i}$ which preserve the $m_{i}$ for $i \neq 0$. Thus, to make the identification $1-1$ we have to consider a quotient by $G \times \mathbb{Z}_{15}$. But the $\mathbb{Z}_{15}$ is already enforced by the projectivization in $\mathbb{P}_{(1,3,3,3,5)}^{4}[15]$. Hence, we are left with $\mathbb{P}_{(1,3,3,3,5)}^{4}[15] / G$ which is the result obtained using the transposition argument.

\section{The Moduli Space of The Mirror}

With the fundamental period at hand we turn to its behavior around the singular points in the moduli space. The computation is by now standard, follows that of [6] and can be found in appendix B. Suffice it to say that we in this way understand the monodromy around $\psi=\infty$.

Our next step is to focus on the complex structure moduli space, and in particular the large complex structure and $\psi=\infty$ limit points. We begin by reviewing the set-up leading to the monomial-divisor mirror map from [27.

In the previous section the toric variety $V=\mathbb{P}(P)$ was described as embedded in $\mathbb{P}^{|P|-1}$. $V$ can also be constructed from the normal fan $\mathcal{N}(P)$ of $P$; in this situation, this is the fan in $N_{\mathbb{R}}$ obtained by taking the union of the cones over all proper faces of $P^{\circ}$. To begin to resolve the singularities of $V$, we can take a simplicial subdivision of $\mathcal{N}(P)$ to arrive at a new fan $\Delta$. It is shown in [32] that one can choose $\Delta$ in such a way that the resulting toric variety $\hat{V}$ (which is an orbifold since the subdivision is simplicial) is projective, and that the edges of the fan are precisely the edges spanned by the points of $\left(P^{\circ} \cap N\right)-\{0\}$.

A key idea leading to the monomial-divisor mirror map is that the points of $P^{\circ} \cap N$ have two interpretations. We have already discussed its interpretation as monomials on $\mathbb{P}\left(P^{\circ}\right)$ (since $N$ plays the role for $P^{\circ}$ that $M$ did for $P$ ). On the other hand, points of $P^{\circ} \cap N-\{0\}$ span edges of $\Delta$, and as such give rise to toric divisors on $\hat{V}$.

A special role is played by the points of $P^{\circ} \cap N$ which are in the interior of a facet. The corresponding divisors of $\hat{V}$ are exceptional for the natural map $\hat{V} \rightarrow V$ and have image equal to a point; hence they are disjoint from the proper transform $\hat{\mathcal{M}}$ of a general

5 We use the notation $\left(\mathbb{Z}_{r}: \Theta_{1}, \Theta_{2}, \Theta_{3}, \Theta_{4}, \Theta_{5}\right)$ for a $\mathbb{Z}_{r}$ symmetry with the action $\left(y_{1}, y_{2}, y_{3}, y_{4}, y_{5}\right) \rightarrow\left(\alpha^{\Theta_{1}} y_{1}, \ldots, \alpha^{\Theta_{5}} y_{5}\right)$, where $\alpha^{r}=1$. 
hyperplane section $\overline{\mathcal{M}}$ of $V$. Let $\left(P^{\circ} \cap N\right)_{0}$ denote the points of $P^{\circ} \cap N$ which do not lie in the interior of a facet.

Now, similarly choose a subdivision $\Delta^{\circ}$ of $\mathcal{N}\left(P^{\circ}\right)$, getting 4 dimensional toric varieties $\hat{U} \rightarrow U$ and hypersurfaces $\hat{\mathcal{W}} \rightarrow \overline{\mathcal{W}}$ analogous to $\hat{V}, V, \hat{\mathcal{M}}$, and $\overline{\mathcal{M}}$.

The monomial-divisor mirror map is the natural isomorphism

$$
H_{\text {poly }}^{2,1}(\hat{\mathcal{W}}) \simeq\left(\left(\mathbb{Z}^{\left(P^{\circ} \cap N\right)_{0}-\{0\}}\right) / M\right) \otimes \mathbb{C} \simeq H_{\text {toric }}^{1,1}(\hat{\mathcal{M}})
$$

The subscripts "toric" and "poly" refer to the subspaces generated by polynomial deformations of $\hat{W}$ and toric divisors of $\hat{\mathcal{M}}$, respectively. See [27] for more details.

The next step is to examine the moduli space of the mirror. According to [27], we let $N^{+}=N \oplus \mathbb{Z}$, and lift the set $\left(P^{\circ} \cap N\right)_{0}$ to the subset of the affine hyperplane $\{(n, 1)\} \subset N^{+}$ which projects to it. We form the secondary fan [32] associated with this set of points. This fan is a finite rational polyhedral subdivision of $H_{\text {toric }}^{1,1}(\hat{\mathcal{M}})$. The toric variety associated with the secondary fan is to be viewed as a "simplified moduli space" for $\hat{W}$, describing all hypersurfaces of the form $\sum c_{i} m_{i}$, where the $m_{i}$ range over the monomials corresponding to points of $\left(P^{\circ} \cap N\right)_{0}$. There is a natural map to the polynomial part of the true moduli space. Assuming that this map is dominant, i.e. has dense image, a precise form of mirror symmetry is conjectured in [27]. We will assume this conjecture to be true, and will use it without verifying dominance. Since our conclusions are consistent with algebraic geometry, we can view this as providing evidence for the conjectured form of mirror symmetry, as well as for dominance.

We now need to interpret this moduli space using the secondary fan. By general toric principles [28], $s$ dimensional cones of the secondary fan correspond to codimension $s$ toric subvarieties of the simplified moduli space.

Note that our one parameter family is determined inside the simplified moduli space by setting the coefficients of all monomials to 0 , except the monomials $m_{0}, \ldots, m_{5}$. Our one parameter family is clearly invariant under the torus (that is, under rescalings of the coefficients), hence corresponds to a codimension 1 cone in the secondary fan. It follows immediately from the standard toric correspondence (see section 3.1 of [28]) that this face is the one spanned by the classes in $H^{1,1}$ of all the exceptional divisors of $\hat{\mathcal{M}}$.

Since the orbifold Kähler cone of $\hat{\mathcal{M}}$ appears in the secondary fan 27] and the hyperplane class $H$ is an edge of the Kähler cone, it follows that $H$ is an edge of the secondary fan. Note: different orbifold Kähler cones can arise from different choices of $\Delta$; but $H$ is an edge of any of these. This is consistent with the discussion following (2.2).

The toric correspondence between edges and divisors (p. 60 of [28]) gives a divisor $D_{H}$ in the simplified moduli space. $D_{H}$ passes through all large complex structure limit points. $D_{H}$ also meets the one parameter family at the point $\psi=\infty$, a point which is invariant under the torus. This point of intersection corresponds (via section 3.1 of [28]) to the cone spanned by the class of $H$ and those of the exceptional divisors. Denote this cone by $\sigma$. 
Suppose that the cardinality of $\left(P^{\circ} \cap N\right)_{0}$ is $n$. Then the secondary fan sits inside $\mathbb{R}^{n-5}$. Ignoring the points $\overrightarrow{0}, v_{1}, \ldots, v_{5}$, we see that there are $n-6$ exceptional divisors $E_{i}$. Let $L \subset H_{\text {toric }}^{1,1}(\mathcal{M}, \mathbb{Z})$ be the lattice spanned by $H$ and the $E_{i}$. As before, let $k$ denote the weight of the hyperplane class of $\mathcal{M}$.

Claim. $L$ is a sublattice of $H_{\text {toric }}^{1,1}(\hat{\mathcal{M}}, \mathbb{Z})$ of index $k$, hence a neighborhood of the point $\psi=\infty$ in the simplified moduli space is given as the quotient of $\mathbb{C}^{n-5}$ by a group of order $k$, as described in section 2.2 of [28].

We will see presently that more is true: the group is in fact $\mathbb{Z}_{k}$.

To see this, we perform the calculation in a convenient choice of coordinates on $H_{\text {toric }}^{1,1}(\hat{\mathcal{M}}, \mathbb{Z})$. Note that elements of $\operatorname{Hom}\left(\mathrm{H}_{\text {toric }}^{1,1}(\hat{\mathcal{M}}, \mathbb{Z}), \mathbb{Z}\right)$ may be thought of as linear dependencies between the points of $\left(P^{\circ} \cap N\right)_{0}$, identified as a subset of $N^{+}$as discussed earlier in this section. In particular, $\overrightarrow{0} \in\left(P^{\circ} \cap N\right)_{0}$ also corresponds to a divisor class $D_{0}$ (but not an effective divisor). Now consider an exceptional divisor $E \subset \hat{\mathcal{M}}$. Since the $v_{i} \operatorname{span} N$, the vector $v \in\left(P^{\circ} \cap N\right)_{0}$ corresponding to $E$ can be expressed as an integral linear combination of the $v_{i}$. Using a superscript of "+" to denote the lifting of a vector to $N^{+}$, there is still a similar expression expressing $v^{+}$as an integral linear combination of $\overrightarrow{0}^{+}$together with the $v_{i}^{+}$. In addition, the relation $\sum_{i} k_{i} v_{i}=0$ gives rise to the relation $\sum_{i} k_{i} v_{i}^{+}-d \overrightarrow{0^{+}}=0$. These linear dependencies clearly span all linear dependencies, and so give rise to coordinates on $H_{\text {toric }}^{1,1}(\hat{\mathcal{M}}, \mathbb{Z})$. Note from the choice of linear dependencies that the exceptional divisors $E_{i}$ have as coordinates the standard unit vectors $e_{i}$ for $1 \leq i \leq n-6$, while $D_{i}$ has last coordinate $k_{i}$. By writing $H=\sum_{i=1}^{5} b_{i} D_{i}+\sum c_{i} E_{i}$ and comparing degrees in $\mathbb{P}_{\left(k_{1}, k_{2}, k_{3}, k_{4}, k_{5}\right)}^{4}$, we see that $k=\sum b_{i} k_{i}$. On the other hand the last coordinate of the right hand side is $\sum b_{i} k_{i}+\sum c_{i} \cdot 0=\sum b_{i} k_{i}$. Hence $H$ has last coordinate $k$. This immediately justifies the claim.

For emphasis, we include a quick example.

Example: Let us consider $H_{\text {toric }}^{1,1}(\hat{\mathcal{M}}, \mathbb{Z})$ for $\mathcal{M} \in \mathbb{P}_{(1,2,2,2,3)}^{4}[10]$. Here we have divisors $D_{1}, \ldots, D_{5}$ corresponding to the proper transforms of $x_{i}=0$, and two exceptional divisors. It is clear from our definitions that in this case (or any example with $k_{1}=1$ ), the vectors $v_{2}, v_{3}, v_{4}, v_{5}$ form a $\mathbb{Z}$ - basis for $N$. In our choices of coordinates we have the following:

$\begin{array}{ccccc}D_{1} & (-2,-2,-2,-3,1) & 0 & 0 & 1 \\ D_{2} & (1,0,0,0,1) & 1 & 1 & 2 \\ D_{3} & (0,1,0,0,1) & 1 & 1 & 2 \\ D_{4} & (0,0,1,0,1) & 1 & 1 & 2 \\ D_{5} & (0,0,0,1,1) & 2 & 1 & 3 \\ E_{1} & (-1,-1,-1,-2,1) & 1 & 0 & 0 \\ E_{2} & (-1,-1,-1,-1,1) & 0 & 1 & 0 \\ D_{0} & (0,0,0,0,1) & -6 & -5 & -10\end{array}$.

The meaning of the above table is that each of the last three columns give coefficients of linear dependencies among the coordinates in the second column. The vectors in the second column are written in the choice of basis for $N_{\mathbb{R}}$ just mentioned, then lifted to $N^{+}$ 
by appending a "1". Note that the entry in the last column after the vector corresponding to $D_{i}$ is $k_{i}$; this is a general fact, arising from the relation $\sum k_{i} v_{i}^{+}-d \overrightarrow{0^{+}}=0$, as mentioned above. The last 3 columns give the coordinates of the divisor class represented by the divisor in the first column. For this example, we calculate $k=6$; the hyperplane class turns out to have coordinates $(4,3,6)$. We will return to this example when we discuss monodromy.

\section{The Yukawa Coupling}

We start by studying the monodromy around the large complex structure point. Knowing this will enable us to choose the relevant flat coordinate, $t$ for which the Yukawa coupling in the $t$-coordinate will coincide with the intersection number computed in section 2 .

\subsection{Monodromy and The Large Complex Structure Limit}

Recall the discussion from the last section. We have a part of the moduli space described by a cone $\sigma$ with edges corresponding to the exceptional divisors and to $H$. In coordinates, these are the standard unit vectors $e_{i}$ for $1 \leq i \leq n-6$ and a vector whose last coordinate is $k$. It now follows from toric generalities [28] that the point of the toric moduli space corresponding to $\sigma$ is singular if $k>1$; in fact, the point is locally the quotient of a polydisc by the group $\mathbb{Z}_{k}$. We will illustrate this by an example presently. Letting $T_{\infty}$ denote the monodromy about this point within the one parameter family, it follows that the monodromy about a general point of $D_{H}$ is $T_{\infty}^{k}$. To see this, standard toric techniques and the above description of the generators of $\sigma$ show that the simplified moduli space is locally an orbifold of the form $\mathbb{C}^{n-5} / \mathbb{Z}_{k}$, where $\mathbb{Z}$ acts by $(x) \mapsto\left(\zeta^{a_{i}} x_{i}\right)_{i}$, where $\zeta=e^{2 \pi i / k}$ and $a_{n-5}=1$, while $D_{H}$ is given in these coordinates by $x_{n-5}=0$ and the one parameter family is given by $x_{1}=\cdots=x_{n-6}=0$. The loop $\left(p_{1}, \ldots, p_{n-6}, e^{2 \pi i \theta}\right)$ where the $p_{i}$ are fixed and non-zero, while $\theta$ ranges from 0 to 1 is seen to go around $D_{H}$ once; but as we let the $p_{i}$ approach 0 , we see that this loops around $D_{H}$ a total of $k$ times. This gives the claimed relation between the various monodromies up to conjugacy, which will not affect the calculation of the Yukawa couplings in the sequel. This will also be illustrated by the following example.

Example. Let us now continue our discussion of a hypersurface $\mathcal{M} \in \mathbb{P}_{(1,2,2,2,3)}^{4}[10]$. We have seen that the toric moduli space is describe by the cone $\sigma$ with edges

$$
(1,0,0),(0,1,0),(4,3,6) \text {. }
$$

(The first two coordinates of the last edge have not been explained here; this is safely done, since the result does not depend on these coordinates.) The toric variety for $\mathbb{C}^{3}$ is described by the cone with edges

$$
(1,0,0),(0,1,0),(0,0,1)
$$


and there is an obvious linear transformation $\mathcal{L}$ mapping the second cone to the first, identifying the first two edges of the first cone with the first two edges of the second cone. But the toric varieties are different, since the integral lattices have changed (the linear transformation used has determinant 6). In other words, the map of tori is not an isomorphism. We are using the standard torus $\left(\mathbb{C}^{*}\right)^{3} \subset \mathbb{C}^{3}$ in identifying the second toric variety with $\mathbb{C}^{3}$ in the usual way. The induced map $\nu$ on the toric varieties, when restricted to the torus, is just

$$
\nu\left(x_{1}, x_{2}, x_{3}\right)=\left(x_{1} x_{3}^{4}, x_{2} x_{3}^{3}, x_{3}^{6}\right) .
$$

This follows because the matrix of $\mathcal{L}$ gives rise to a map between the spaces of one parameter subgroups associated to the toric varieties; we must transpose this matrix to get the map between the associated spaces of characters, and this immediately gives (5.3). The group we must quotient out by is evidently $\nu^{-1}(1,1,1)$, which is the group $\left(\mathbb{Z}_{6}: 2,3,1\right)$.

In the $\left(x_{1}, x_{2}, x_{3}\right)$ coordinates just introduced, the divisor $D_{H}$ corresponds to $x_{3}=0$. But $x_{3}$ is only a multi-valued function on the moduli space. Near a general point of $D_{H}$, we have that $x_{1}$ and $x_{2}$ are non-zero; so $D_{H}$ has $\left(\mathbb{Z}_{6}\right.$ invariant) equation $x_{1} x_{2} x_{3}=0$. Now look at the loop $\theta \mapsto\left(x_{1}, x_{2}, e^{2 \pi i \theta}\right)$. This clearly loops around $x_{1} x_{2} x_{3}=0$ once. But as $x_{1}$ and $x_{2}$ approach 0 , we must take $x_{3}^{6}$ as our invariant equation; that is, the loop winds around 6 times, illustrating the general situation.

We now know that $T_{\infty}^{k}$ gives the monodromy around the large complex structure limit point. On general grounds the Picard-Fuchs equation satisfied by $\varpi_{0}$ has at $\psi=\infty$ a quasi-unipotent point of index $l$ [22]. By this we mean that there exists a natural number $k$, defined as above, such that

$$
U^{l-1} \neq 0, \quad U^{l}=0, \quad \text { where } U=T_{\infty}^{k}-I
$$

It was observed that in fact the Yukawa coupling in the large complex structure limit can be read off from $U^{3}$ [8] and hence that $l=4.6$ In an integral and symplectic basis one can show that $U^{3}=y_{\psi \psi \psi}^{\text {lcs }} E$ where $E$ is the zero-matrix except for one entry along the first column which is 1 [8]. In our basis, $U^{3}$ has more than one non-vanishing entry in the first column. However, by using that we know two of the basis elements in the integral basis one can show that the smallest (in terms of absolute value) of the non-zero entries in $U^{3}$ gives the Yukawa coupling, $y_{\psi \psi \psi}^{\mathrm{lcs}}$.

Thus, from (5.4) it follows that the Yukawa coupling is readily computed for all of the 7,555 transverse hypersurfaces in weighted $\mathbb{P}^{4}$. All we have to do is to calculate $U^{3}$. However, there is a slight computational problem in that there are models for which $b_{3} \sim O\left(10^{3}\right)$ [4], i.e. the number of periods is $O\left(10^{3}\right)$. Combined with the fact that for some of these models $k \sim O\left(10^{8}\right)$, merely computing $T_{\infty}^{k}$ would take quite some time! Fortunately, the problem is very much simplified because of the special form that the matrices $T$ and $A$, and hence $T_{\infty}$, take, see (B.17) and table B.1. The first step is to put

6 Thus, based on our explicit calculation, discussed below, we conjecture that any Picard-Fuchs equation with solutions $\varpi_{i}$ as given by (B.7) have a quasi-unipotent point, $\psi=\infty$, of index 4 . 
$T_{\infty}$ on a block-diagonal form. This not only makes the computation feasible in real time but also shows that the Yukawa coupling can be written as

$$
y_{\psi \psi \psi}^{\mathrm{lcs}}=k^{3} \frac{C_{1}}{C_{2}}
$$

where the $C_{i}$ can be found in terms of the basis which (block) diagonalizes $U$ It turns out that, in this particular situation, to compute $C_{i}$ is an $O\left(N^{2}\right)$ process for an $N \times N$ matrix while matrix multiplication is $O\left(N^{3}\right)$. This is what saves the day and makes it possible to put the problem on a computer. The calculation has been performed for some 7,400 models. For the remaining ones it has not yet been possible to construct the matrix $A$ associated to the phase symmetry $\mathcal{A}$. However, we believe that this is merely a technical problem and that within the near future all couplings will be found by this method.

\subsection{The Flat Coordinate and The Yukawa Coupling}

We now turn to studying the flat coordinate, $t$ which will provide the mirror map between $\mathcal{M}$ and $\mathcal{W}$. We then go on to give the general expression for the Yukawa coupling $y_{\psi \psi \psi}$ as a function of $\psi$ for $\mathcal{W}$. Knowledge of $t$, and hence the mirror map, enable us to map $y_{\psi \psi \psi}$ to $y_{t t t}$ where $y_{t t t}$ is the Yukawa coupling on $\mathcal{M}$ corresponding to the Kähler deformation.

The naïve flat coordinate, relevant for the $\psi \rightarrow \infty$ limit is defined by [6]

$$
T_{\infty}: \tilde{t} \rightarrow \tilde{t}+1
$$

However, from the previous discussion we know that the monodromy around the large complex structure limit point is given by $T_{\infty}^{k}$. Hence, the natural flat coordinate from that point of view is

$$
T_{\infty}^{k}: t \rightarrow t+1, \quad t=\tilde{t} / k
$$

In fact $t=\tilde{t} / k$ is the analog of $\rho^{*}(H)=k \tilde{H}$ where $\tilde{H}$ is the hyperplane class induced from $\mathbb{P}^{4}$ and $H \in H^{2}(\mathcal{M}, \mathbb{Z})$ as discussed in appendix A. From (5.6) and (5.7) we can find $t$ as a linear combination of the periods $\varpi_{j}$ given by (B.7). By analytically continuing the $\varpi_{j}$ to large $\psi, t(\psi)$ will give the mirror map. Although this procedure can straightforwardly be carried out along the lines of [6] we are only interested in the large $\psi$ behavior of $t(\psi)$. This is obtained by studying the Riemann $\mathcal{P}$-symbol associated to the generalized hypergeometric equation to which the $\varpi_{j}$ are the solutions. In fact by looking at (3.4) we readily read off that among the solutions as $\psi \rightarrow \infty$ there are four of the type $\left(1, \log \left((\psi d)^{d}\right), \log ^{2}\left((\psi d)^{d}\right), \log ^{3}\left((\psi d)^{d}\right)\right)$. Thus, using this and (5.7),

$$
t \sim-\frac{1}{k} \frac{d}{2 \pi i} \log (\psi d) \quad \psi \rightarrow \infty .
$$

Since $t$ is the coordinate on the Kähler moduli space and $\psi$ parametrizes the fundamental deformation on the complex structure, eq. (5.8) gives the mirror map for large $\psi$.

7 In the next section we will show how to compute $C_{1} / C_{2}$ independently for all models. 
In 20,6] it was shown that the Yukawa coupling on the space of complex structure deformations is given by

$$
\begin{aligned}
y_{\psi \psi \psi} & =\int \Omega \wedge \frac{\partial^{3} \Omega}{\partial \psi^{3}} \\
& =z^{a}\left(\frac{d}{d \psi}\right)^{3} \mathcal{G}_{a}-\mathcal{G}_{a}\left(\frac{d}{d \psi}\right)^{3} z^{a},
\end{aligned}
$$

where we restrict to the fundamental deformation. The $\left(\mathcal{G}_{a}, z^{b}\right)$ are the components of an integral and symplectic basis. In particular, $\mathcal{G}_{0}=\varpi_{0}$ and $z^{0}=\varpi_{0}-\varpi_{1}$ [6]. Knowing these two basis vectors is what makes it possible to make a (partial) change of basis from the $\varpi_{j}$ to the $\left(z^{a}, \mathcal{G}_{a}\right)$ and hence to read off $y_{\psi \psi \psi}^{\text {lcs }}$ from $U^{3}$ as discussed in the previous subsection. Unfortunately, it does not seem to be possible to construct the complete symplectic basis with only the knowledge of the conifold singularity.

The Yukawa couplings can also be found by studying the chiral ring of the underlying $N=2$ superconformal field theory [33]. The chiral ring is equivalent to the ring of monomials based on the defining equation, $\hat{p}_{\psi}$, of $\mathcal{W}$ modulo the ideal generated by $\mathrm{d} \hat{p}_{\psi}$. On general grounds it has been shown that the unnormalized coupling is given by [34]

$$
y_{\psi \psi \psi}=\frac{\left(\prod_{i=1}^{5} y_{i}\right)^{3}}{\mathcal{H}}
$$

where $\mathcal{H}$, the Hessian, is the determinant of the matrix of second derivatives of $\hat{p}_{\psi}$. In general, one would have to use the ring to rewrite $\left(\prod_{i=1}^{5} y_{i}\right)^{3}$ to a monomial proportional to $\mathcal{H}$. This would necessarily involve knowing the explicit form of $\hat{p}_{\psi}$. However, we will argue that one can find $y_{\psi \psi} \psi$ without explicitly referring to the $\hat{p}_{\psi}$. Recall that in general there does not exist a representation of $\mathcal{W}$ as a hypersurface in a weighted projective space although $\mathcal{W}$ is always well defined as a hypersurface in a toric variety.

The first point to observe is that because of the conifold singularity at $\psi=$ $\left(\prod_{i=1}^{5} k_{i}^{k_{i} / d}\right)^{-1}$ the coupling will also have such a singularity. This can be understood by looking at eq. (5.9) . The periods in the symplectic basis are linear combinations of the $\varpi_{j}$ which have conifold singularities. Hence, the denominator in (5.10) must have the form $\left(1-\prod_{i=1}^{5} k_{i}^{k_{i}} \psi^{d}\right)$, up to an overall numerical factor.

The next point is to use that we know $y_{\psi \psi \psi}$ in the large complex structure limit from the monodromy calculation in the previous section. In terms of the flat coordinate and the large complex structure gauge [6] the coupling is given by

$$
y_{t t t}=\frac{y_{\psi \psi \psi}}{\varpi_{0}^{2}}\left(\frac{d \psi}{d t}\right)^{3} .
$$

From (5.8) we have $\left(\frac{d \psi}{d t}\right) \sim-k \frac{2 \pi i}{d} \psi$. Thus, in order for (5.11) to be consistent we find that

$$
y_{\psi \psi \psi}=\left(\frac{d}{2 \pi i}\right)^{3} \frac{y_{\psi \psi \psi}^{\mathrm{lcs}}}{k^{3}} \frac{\prod_{i=1}^{5} k_{i}^{k_{i}} \psi^{d-3}}{\left(1-\prod_{i=1}^{5} k_{i}^{k_{i}} \psi^{d}\right)} .
$$


Because of the mirror map, (5.8) and (5.11) and by expanding (5.12) around large $\psi$, $y_{\psi \psi \psi}^{\text {lcs }}$ is predicted to give the $(1,1)$ form Yukawa coupling in the large radius limit on $\mathcal{M}$, i.e. the intersection number $y_{J J J}$ calculated in section 2. Note that in terms of the flat coordinate $\tilde{t}$, the Yukawa coupling as $\psi \rightarrow \infty$ is $y_{\psi \psi \psi}^{\text {lcs }} / k^{3}$. This is the lowest order term in the instanton expansion as computed for some examples in [8].

Let us now show how we in fact can compute $y_{\psi \psi \psi}^{\text {lcs }}$ analytically. From [6] an integral symplectic basis can be chosen such that under monodromy around the conifold, $\left(\mathcal{G}_{i}, z^{i}\right) \rightarrow\left(\mathcal{G}_{i}+\delta_{i} z^{0}, z^{i}\right)$. Thus, only $\mathcal{G}_{0}$ diverges logarithmically as we approach the singularity (B.14). It then follows that in computing $y_{\psi \psi \psi}$ from (5.9) in the limit $\psi \rightarrow\left(\prod_{i=1}^{5} k_{i}^{k_{i} / d}\right)^{-1}$ the leading term comes from $z^{0}\left(\frac{d}{d \psi}\right)^{3} \mathcal{G}_{0}$. From (B.14) the $\varpi_{i}$ are appropriate for studying the conifold singularity and hence it is a straightforward, though a bit tedious, exercise to obtain $y_{\psi \psi \psi}$ as $\psi \rightarrow\left(\prod_{i=1}^{5} k_{i}^{k_{i} / d}\right)^{-1}$. We find, using (5.9), that the leading behavior of $y_{\psi \psi \psi}$ as we approach the conifold singularity is given by

$$
y_{\psi \psi \psi} \sim\left(\frac{d}{2 \pi i}\right)^{3} \frac{d}{\prod_{i=1}^{5} k_{i}}\left(\prod_{i=1}^{5} k_{i}^{k_{i} / d}\right)^{3} \sum_{N}\left(\psi \prod_{i=1}^{5} k_{i}^{k_{i} / d}\right)^{d N} .
$$

Comparing with (5.12) by taking the conifold limit we readily read off

$$
y_{\psi \psi \psi}^{\mathrm{lcs}}=\frac{d k^{3}}{\prod_{i=1}^{5} k_{i}} .
$$

Thus, we get perfect agreement with (2.4)!

To illustrate the ideas explained above let us now return to the examples from section 2 .

Example 1. Consider the hypersurface $\mathcal{M}_{1} \in \mathbb{P}_{(1,1,1,2,2)}^{4}[7]_{-186}^{2,95}$ defined by $p_{1}=0$ where $p_{1}$ is a transverse polynomial. (As usual the sub- and superscripts refer to $\chi_{E}$ and $b_{1,1}, b_{2,1}$ respectively on a Calabi-Yau desingularization of $\mathcal{M}_{1}$.) From (3.3) the period corresponding to the fundamental deformation $\prod_{i=1}^{5} y_{i}$ on the mirror manifold $\mathcal{W}_{1}$ is

$$
\varpi_{0}=\sum_{m=0}^{\infty} \frac{\Gamma(7 m+1)}{\Gamma^{2}(2 m+1) \Gamma^{3}(m+1)(7 \psi)^{7 m}}
$$

From (B.16) we have $c_{j}=(1,1,-2,-1,4,-1,-2)$. The number of linearly independent

8 To be more precise, one has to study the Riemann $\mathcal{P}$-symbol associated to the differential equation to which the periods are solutions. One then finds that $\varpi_{j} \sim\left(\psi-\left(\prod_{i=1}^{5} k_{i}^{k_{i} / d}\right)^{-1}\right)^{l_{j}}$, $l_{j} \in N$ for all periods except one, $\mathcal{G}_{0} \sim z^{0} \log \left(\psi-\left(\prod_{i=1}^{5} k_{i}^{k_{i} / d}\right)^{-1}\right)$ with $z^{0} \sim\left(\psi-\left(\prod_{i=1}^{5} k_{i}^{k_{i} / d}\right)^{-1}\right)$ as $\psi \rightarrow\left(\prod_{i=1}^{5} k_{i}^{k_{i} / d}\right)^{-1}$. 
periods is $7-1=6$ since there is only one relation among the $\varpi_{j} .9$ From

$$
\sum_{j=0}^{6} \varpi_{j}=0
$$

and the $c_{j}$ 's we get the monodromy matrices in table 5.1.

$$
A=\left(\begin{array}{rrrrrr}
0 & 1 & 0 & 0 & 0 & 0 \\
0 & 0 & 1 & 0 & 0 & 0 \\
0 & 0 & 0 & 1 & 0 & 0 \\
0 & 0 & 0 & 0 & 1 & 0 \\
0 & 0 & 0 & 0 & 0 & 1 \\
-1 & -1 & -1 & -1 & -1 & -1
\end{array}\right) \quad T=\left(\begin{array}{rrrrrr}
2 & -1 & 0 & 0 & 0 & 0 \\
1 & 0 & 0 & 0 & 0 & 0 \\
-2 & 2 & 1 & 0 & 0 & 0 \\
-1 & 1 & 0 & 1 & 0 & 0 \\
4 & -4 & 0 & 0 & 1 & 0 \\
-1 & 1 & 0 & 0 & 0 & 1
\end{array}\right)
$$

Table 5.1: Monodromy matrices $(A, T)$ associated to $\psi=0,\left(2^{2} 2^{2}\right)^{-1 / 7}$ for $\mathcal{W}_{1}$.

As noted in Example 1 of section 2, by (A.3) we have $k=2$. The monodromy calculation then gives

$$
U=T_{\infty}^{2}-I, \quad U^{3}=14 E
$$

Thus, by the argument in section $5.1 y_{\psi \psi \psi}^{\mathrm{lcs}}=14$. Now comparing with the intersection calculation in section 2, we see that the couplings indeed agree.

Example 2. Let $p_{2}=0$, where $p_{2}$ is a transverse polynomial, define a hypersurface $\mathcal{M}_{2} \in$ $\mathbb{P}_{(1,1,3,4,6)}^{4}[15]_{-198}^{7,106}$. From $(3.3)$ the period corresponding to the fundamental deformation $\prod_{i=1}^{5} y_{i}$ on the mirror manifold $\mathcal{W}_{2}$ is

$$
\varpi_{0}=\sum_{m=0}^{\infty} \frac{\Gamma(15 m+1)}{\Gamma^{2}(m+1) \Gamma(3 m+1) \Gamma(4 m+1) \Gamma(6 m+1)(15 \psi)^{15 m}} .
$$

From (B.16) we have $c_{j}=(1,1,-1,0,-1,0,1,-1,2,-1,1,0,-1,0,-1)$ and there will be $15-3=12$ linearly independent periods 10 Thus, in this case we do not obtain all of the

9 Note that on $\mathcal{W}_{1}, 2 b_{2,1}+2=6$ and we get all of the periods through the action of the phase symmetry $\mathcal{A}$. In general this will not be the case. The missing periods are the complex deformations which cannot be written as polynomial deformations [35].

10 From (B.8) we see that $k_{3}$ will result in three constraints on the $\varpi_{j}$ of the form $\sum_{j=0}^{4} \varpi_{l+3 j}=$ 0 where $l=0,1,2$. There will be no new restrictions from the other $k_{i}$. 
periods. This is a reflection of the fact that only five of the total seven complex deformations are given as polynomial deformations. This may provide important information in constructing $\mathcal{W}_{2}$. From the $c_{j}$ 's above and the relation $\sum_{j=0}^{4} \varpi_{3 j}=0$ one easily finds the relevant monodromy matrices, see table 5.2 . We find that $k=12$ and so

$$
U=T_{\infty}^{12}-I \quad U^{3}=360 E .
$$

Just as for the first example we conclude that $y_{\psi \psi \psi}^{\mathrm{lcs}}=360$. Comparing with the calculation done on $\mathcal{M}_{2}$ the couplings agree.

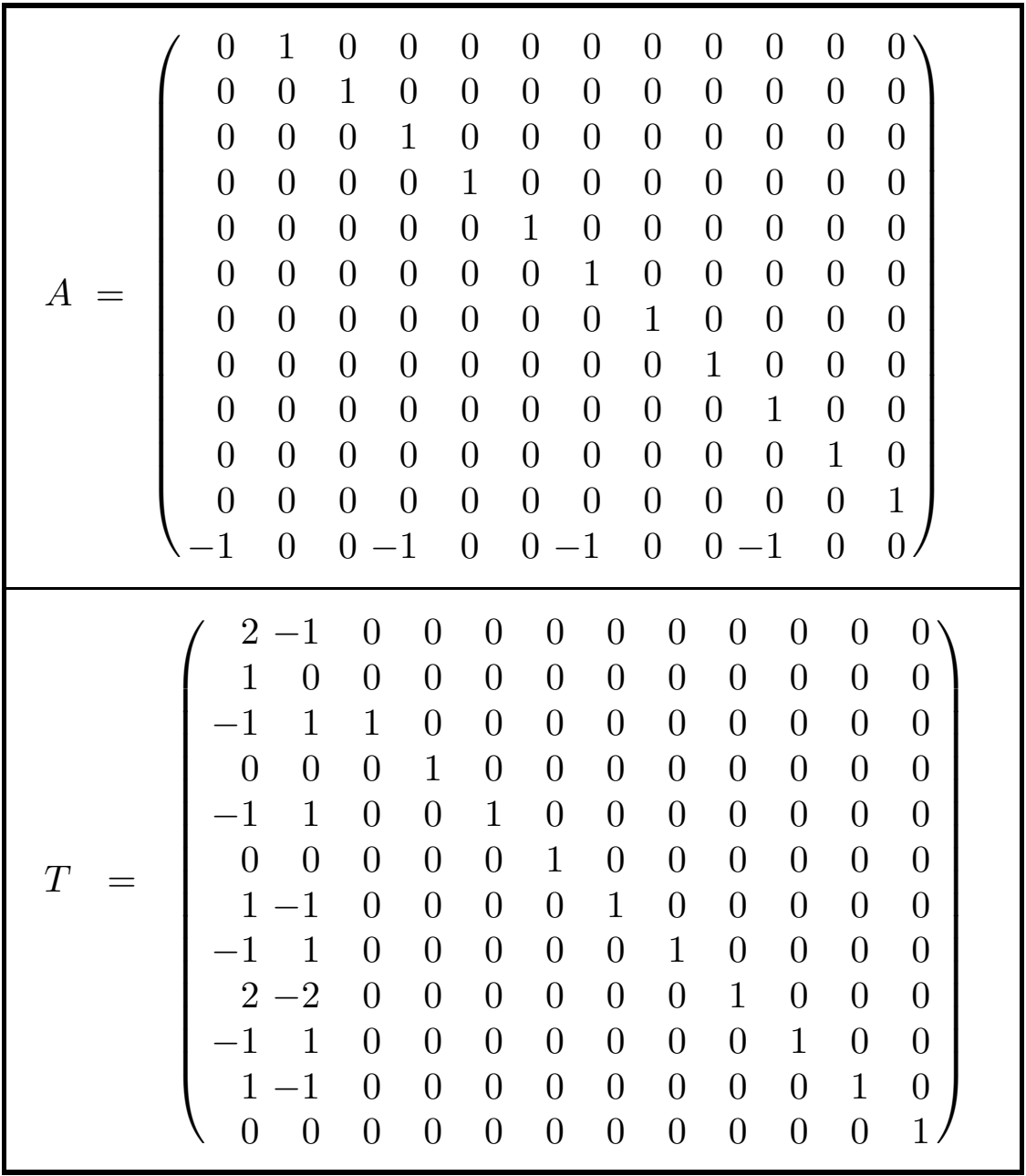

Table 5.2: Monodromy matrices $(A, T)$ associated to $\psi=0, \quad\left(3^{3} 4^{4} 6^{6}\right)^{-1 / 15}$ for $\mathcal{W}_{2}$.

In comparing the topological couplings computed on the 7,555 models listed in [4] with $y_{\psi \psi \psi}^{\mathrm{lcs}}$ obtained through monodromy considerations described above we find complete agreement; though for a few of the models $y_{\psi \psi \psi}^{\mathrm{lcs}}$ remains to be calculated. This gives a very large set of verifications of mirror symmetry, although only to lowest order. The 
toric topological couplings have been computed in principle for toric hypersurfaces up to a constant [36]; our work fixes the constant, verifying more of the conjectured mirror symmetry in this situation. In fact, we have shown that our methods apply to a larger class of models than we have discussed above, by checking agreement between the couplings on $\mathcal{M}$ and $\mathcal{W}$ for some non-transverse spaces considered in [29].

In the above we have used the monomial-divisor mirror map [27] in finding $k$. This in turn was used to show that the relevant monodromy is $T_{\infty}^{k}$ around the large complex structure limit point. However, the actual numerical computation does not rely on these facts. In a case by case study one finds that $T_{\infty}$ has eigenvalues which are $k$ th roots of unity. From this and the general discussion in [8] regarding the Yukawa couplings in the large complex structure limit we deduce that the monodromy around the large complex structure limit point indeed is $T_{\infty}^{k}$. The definition of the flat coordinate $t$ then naturally follows. Thus all the ingredients necessary for computing $y_{\psi \psi \psi}^{\mathrm{lcs}}$ can be found without relying on the toric picture in general and the monomial-divisor mirror map in particular. It is very reassuring though, that the two approaches agree and hence our explicit calculation lends further support to some of the conjectures made in [27.

\section{Discussions}

In this paper we have shown the agreement between the $(1,1)$ form Yukawa coupling on $\mathcal{M}$ and the $(2,1)$ form coupling computed on its mirror partner $\mathcal{W}$ in the large radius and complex structure limit respectively, restricting to a particular one-dimensional subspace of the moduli space. On the one hand this confirms mirror symmetry, in a particular limit, for a large class of manifolds. On the other hand the formulae for the respective coupling are very similar. This may indicate that we ought to be able to formulate the computation only in terms of the Kähler modulus. Unfortunately, although special geometry applies as well for the Kähler class, very little is known about how we would carry out such a calculation in practice.

The computation also shows that it is possible to find the Yukawa couplings and part of the modular group without knowing the integrable basis, or at least with very little knowledge of it. This basis is in general hard to construct and hence it is gratifying that detailed information about the moduli space still can be obtained.

Finally (and this was one of our original motivations), we may view the calculation as really being a calculation for a pair of mirror families, namely $\mathcal{M} \in \mathbb{P}_{\left(k_{1}, k_{2}, k_{3}, k_{4}, k_{5}\right)}^{4}[d]$ which has just one Kähler modulus, and the one parameter fundamental deformation of $\mathcal{W}$ which we have been considering. Much of the toric calculation was used merely to justify the intuitive assertion that since we have to multiply the naive Kähler class by $k$ to get an integral class, we must raise the naive monodromy to the power $k$ to correctly scale the flat coordinate for the fundamental deformation. This complication arises because we are dealing with Calabi-Yau orbifolds rather than manifolds. The other main conclusion of the toric calculation is the local description of the moduli space near $\psi=\infty$. While we used the methods of [27] for this, we could have reached the desired conclusion without this. 
The advantage of organizing our calculations in this way is to also bring out the point that we have explicitly checked some consequences of the conjectures made in [27], as well as to make more precise the conclusions of [36].

Acknowledgements: It is a pleasure to thank P. Candelas, X. de la Ossa, T. Hübsch, F. Liu, C. Myers and in particular D. Morrison for useful discussions. P. B. was supported by the American-Scandinavian Foundation, the Fulbright Program, NSF grants PHY 8904035 and PHY 9009850, DOE grant DE-FG02-90ER40542 and the Robert A. Welch Foundation. S. K. was supported by NSF grant DMS-9311386. P. B. would also like to thank the ITP, Santa Barbara and the Theory Division, CERN for their hospitality where part of this work was carried out. 


\section{Appendix A. The Hyperplane Class}

Let us now turn to discuss the hyperplane class $H$ of a weighted hypersurface $\mathcal{M}$ in $\mathbb{P}_{\left(k_{1}, k_{2}, k_{3}, k_{4}, k_{5}\right)}^{4}$. The map $\rho: \mathbb{P}^{4} \rightarrow \mathbb{P}_{\left(k_{1}, k_{2}, k_{3}, k_{4}, k_{5}\right)}^{4}$ induces an inclusion $\rho^{*}: H^{2}(\mathcal{M}, \mathbb{Z}) \rightarrow$ $H^{2}(X, \mathbb{Z})$ with $X \subset \mathbb{P}^{4}$. Let $\tilde{H} \in H^{2}(X, \mathbb{Z})$ be the hyperplane class induced from the one on $\mathbb{P}^{4}$. Let $H \in H^{2}(\mathcal{M}, \mathbb{Z})$ be the unique generator with the property that $\rho^{*}(H)$ is positive. Suppose that $\rho^{*}(H)=k \tilde{H}$. We summarize this situation by saying that $k$ is the weight of the hyperplane class $H$ of $\mathcal{M} . k$ is all that is needed to calculate the intersection numbers that we need. In fact:

$$
H^{3}=\frac{k^{3} d}{\prod_{i=1}^{5} k_{i}} .
$$

(Compare with [9]).

To see this, note that since $\rho$ is finite of degree $\prod_{i=1}^{5} k_{i}$, we have

$$
H^{3}=\frac{1}{\prod_{i=1}^{5} k_{i}}\left(\rho^{*}(H)\right)^{3}=\frac{k^{3} d}{\prod_{i=1}^{5} k_{i}},
$$

the last equality holding since the generator of $H^{2}(X, \mathbb{Z})$ has degree $d$ (i.e. $X$ has degree d).

It remains to find the weight $k$ of $H$. Equivalently, this is the smallest positive integer with the property that the restriction to $\mathcal{M}$ of forms of degree $k$ are identified with the sections of a line bundle. It suffices to show this when the forms are restricted to each of a collection of open subsets of $\mathcal{M}$, which taken together cover $\mathcal{M}$. Equivalently, there must exist on sufficiently small open sets a nowhere vanishing holomorphic form of degree $k$ (which is equivalent to giving a trivialization of the bundle). For generalities on the pathologies that can occur, see [37].

We first illustrate with a simple example, $\mathbb{P}_{(1,1,2,2,2)}^{4}[8]_{-168}^{2,86}[\mathbb{8}]$. By a rational monomial, we mean an expression $\prod_{i} x_{i}^{r_{i}}$, where $r_{i}$ is an integer (allowed to be negative). We claim that $k=2$. First note that $k=1$ does not suffice. To see this, first note that the only rational monomials of degree 1 must contain terms involving $x_{0}$ and/or $x_{1}$ in the numerator or denominator. These monomials cannot be both holomorphic and nowhere vanishing along the locus $x_{0}=x_{1}=0$, which is a plane quartic curve. On the other hand, for $k=2$, we consider the open cover $U_{i}=\left\{\left(x_{1}, \ldots, x_{5}\right) \mid x_{i} \neq 0\right\}$, and exhibit the respective degree 2 forms $x_{1}^{2}, x_{2}^{2}, x_{3}, x_{4}, x_{5}$ which are holomorphic and nowhere vanishing in the respective open sets $U_{1}, \ldots, U_{5}$.

Another general feature exhibited by this example is that the problem occurs along the singular locus (the plane quartic), which has quotient singularities of order 2. As we shall see presently, $k=2$ can be derived from this fact.

We now turn to the general case. First of all, if none of the $x_{i}$ are zero (so that we are situated in the smooth locus), then any degree suffices locally: $\operatorname{since} \operatorname{gcd}\left(k_{1}, \ldots, k_{5}\right)=1$, a rational monomial of degree 1 can be constructed, and any such monomial may be used 
to trivialize a bundle. So the only problems can occur at points at which at least one of the coordinates is 0 .

Without loss of generality, assume that we are at a point where $x_{1}=x_{2}=\ldots=x_{s}=0$ while all other coordinates are non-zero. We must find a rational expression of degree $k$ with neither zeros nor poles at this point. If such an expression exists, we can substitute $x_{1}=x_{2}=\ldots=x_{s}=0$ into it to get another. In this way, we may assume that such an expression only involves $x_{s+1}, \ldots, x_{5}$. For this to be possible, the gcd of $k_{s+1}, \ldots, k_{5}$ must divide $k$. Conversely, if the gcd of $k_{s+1}, \ldots, k_{5}$ divides $k$, then a degree $k$ rational monomial can be constructed from the last $5-s$ coordinates, and such a monomial is holomorphic and non-vanishing in a neighborhood of the point in question.

Now we investigate which combinations of coordinates can be zero for some point of $\hat{\mathcal{M}}$. This is easy. Setting three or fewer coordinates to 0 gives a positive dimensional subspace of $\mathbb{P}_{\left(k_{1}, k_{2}, k_{3}, k_{4}, k_{5}\right)}^{4}$, hence the hypersurface $\hat{\mathcal{M}}$ must have non-empty intersection with it. On the other hand, if four coordinates are set to zero (say the first 4), then this determines the unique point $(0,0,0,0,1)$ of $W \mathbb{P}^{4}$. This point is in $\hat{\mathcal{M}}$ (we assume $\hat{\mathcal{M}}$ is general) if and only if every degree $k$ monomial involves at least one of the first 4 coordinates, or equivalently, there is no monomial involving only $x_{5}$. This is equivalent to $d$ not being a multiple of $k_{5}$. Thus, we see that $k$ is given by

$$
k=\operatorname{lcm}\left(\left\{\operatorname{gcd}\left(k_{i}, k_{j}\right) \mid i \neq j \forall i, j\right\} \cup\left\{k_{i} \mid \quad k_{i} \text { does not divide } d\right\}\right) .
$$

Alternatively, one can use the notion of Cartier divisors rather than line bundles. The point is that the zero locus of a form of degree $k$ cannot be a Cartier divisor near a point of the singular locus unless its degree is a multiple of the index of the singular point. By the index, we mean the order of the isotropy group of a point of $X$ lying over the point in question. This is the approach taken in a similar calculation appearing in [9].

It is easy to see that the calculation works out exactly as above. The index of a point where $x_{1}=\ldots=x_{s}=0$ is just the gcd of $k_{s+1}, \ldots, k_{5}$ as before, as one easily computes. This includes smooth points, which have index 1.

\section{Appendix B. Monodromy}

In what follows we will use the technique developed in [6] to obtain the monodromy matrices around the singular points in the modular space of $\psi$-deformations. For further details we refer the reader to [6].

By studying the condition under which $\varpi_{0}$ converges one finds that there is a conifold singularity 11 given by

$$
1-\prod_{i=1}^{5} k_{i}^{k_{i}} \psi^{d}=0
$$

11 Strictly speaking we would have to compute the matrix of second derivatives of $\hat{p}_{\psi}$ to show that it is not singular where $\hat{p}_{\psi}=\mathrm{d} \hat{p}_{\psi}=0$. For now we will assume that this is a singularity of conifold type and return to this question as we discuss the monodromy around the singularity. 
In order to find the monodromy around this singularity we need to analytically continue (3.1) to small $\psi$. Let us assume that $k_{1}$ is the smallest weight. By using the multiplication formula,

$$
\Gamma\left(k_{1} n+1\right)=n k_{1}^{k_{1} n+1 / 2}(2 \pi)^{\left(1-k_{1}\right) / 2} \prod_{r=0}^{k_{1}-1} \Gamma\left(n+\frac{r}{k_{1}}\right)
$$

we can rewrite $(3.1)$ as

$$
\begin{aligned}
\varpi_{0} & =(2 \pi)^{\left(k_{1}-1\right) / 2} k_{1}^{-1 / 2} \\
& \times \sum_{n=0}^{\infty} \frac{\Gamma(d n+1)}{\Gamma(n+1) \prod_{r=1}^{k_{1}-1} \Gamma\left(n+\frac{r}{k_{1}}\right) \prod_{i=2}^{5} \Gamma\left(k_{i} n+1\right)\left(k_{1}^{k_{1}}(d \psi)^{d}\right)^{n}} .
\end{aligned}
$$

To analytically continue $\varpi_{0}$ to small $\psi$ we use Barnes integral to write $(\mathbb{B} .3)$ as

$$
\varpi_{0}=\frac{(2 \pi)^{\left(k_{1}-1\right) / 2}}{2 \pi i k_{1}^{1 / 2}} \int_{\gamma} \mathrm{d} s \frac{\Gamma(-s) \Gamma(d s+1) e^{i \pi s}\left(k_{1}^{k_{1} / d} d \psi\right)^{-d s}}{\prod_{r=1}^{k_{1}-1} \Gamma\left(s+\frac{r}{k_{1}}\right) \prod_{i=2}^{5} \Gamma\left(k_{i} s+1\right)} .
$$

By closing $\gamma$, given by $s=-\epsilon+i y$, where $y \in(-\infty,+\infty)$ and $0<\epsilon<1 / d$, to the right and demanding $|\psi|>\left(\prod_{i=1}^{5} k_{i}^{k_{i} / d}\right)^{-1}$ we recover $(\mathrm{B} .3)$ due to the poles in $\Gamma(-s)$. However, there are also poles for $d s+1=-m$ with $m=0,1, \ldots$ and by requiring that $|\psi|<\left(\prod_{i=1}^{5} k_{i}^{k_{i} / d}\right)^{-1}$ we can encircle the latter poles by closing the contour to the left,

$$
\begin{aligned}
\varpi_{0} & =-\frac{(2 \pi)^{\left(k_{1}-1\right) / 2}}{k_{1}^{1 / 2} d} \\
& \times \sum_{m=1}^{\infty} \frac{\Gamma\left(\frac{m}{d}\right) \alpha^{\frac{d-1}{2} m}\left(k_{1}^{k_{1} / d} d \psi\right)^{m}}{\Gamma(m) \prod_{r=1}^{k_{1}-1} \Gamma\left(\frac{r}{k_{1}}-\frac{m}{d}\right) \prod_{i=2}^{5} \Gamma\left(1-\frac{k_{i} m}{d}\right)} .
\end{aligned}
$$

So far we have only considered one period. But we know on general grounds that $\varpi_{0}$ satisfies a generalized hypergeometric equation of order $q$ to which there are $q$ linearly independent solutions. To construct the other $q-1$ solutions we use that the generator of the phase symmetry in the modular group is given by

$$
\mathcal{A}: \psi \rightarrow \alpha \psi \quad, \quad \alpha^{d}=1 \text {. }
$$

This follows from $\Omega(\alpha \psi)=\Omega(\psi)[6]$, i.e. the theory is invariant under the action of $\mathcal{A}$. So $\varpi_{0}(\alpha \psi)$ must also be a solution to the Picard-Fuchs equation. Hence, we obtain the other periods by acting with $\mathcal{A}$,

$$
\begin{aligned}
\varpi_{j}(\psi) & \stackrel{\text { def }}{=} \varpi_{0}\left(\alpha^{j} \psi\right)=-\frac{(2 \pi)^{\left(k_{1}-1\right) / 2}}{k_{1}^{1 / 2} d} \\
& \times \sum_{m=1}^{\infty} \frac{\Gamma\left(\frac{m}{d}\right) \alpha^{\left(\frac{d-1}{2}+j\right) m}\left(k_{1}^{k_{1} / d} d \psi\right)^{m}}{\left.\Gamma(m) \prod_{r=1}^{k_{1}-1} \Gamma\left(\frac{r}{k_{1}}-\frac{m}{d}\right)\right) \prod_{i=2}^{5} \Gamma\left(1-\frac{k_{i} m}{d}\right)} .
\end{aligned}
$$


Note that the $\varpi_{j}$ are not all linearly independent. This is seen by observing that the denominator has poles for

$$
\frac{m k_{i}}{d}=1,2, \ldots \text { and } \frac{m}{d}-\frac{r}{k_{1}}=0,1, \ldots
$$

In particular we have the relation

$$
\sum_{j=0}^{d-1} \varpi_{j}=0
$$

as one relation but depending on the weights $k_{i}$ there will in general be more. By using relations of the type $(\mathbb{B . 9})$ we can write down the matrix, $A$, associated to the action of $\mathcal{A}$. Then, defining the period vector as

$$
\varpi=\left(\begin{array}{c}
\varpi_{0} \\
\varpi_{1} \\
\vdots \\
\varpi_{q-1}
\end{array}\right),
$$

the action of $\mathcal{A}$ is simply given by (see eq. $(\mathbb{B} .6)$ )

$$
\mathcal{A}: \varpi_{j} \rightarrow \varpi_{j+1}, \quad j=0, \ldots, q-1 .
$$

But $\varpi_{q}$ is not one of the components of $\varpi$. However,

$$
\varpi_{q}=\sum_{j=0}^{q-1} a_{j} \varpi_{j}
$$

where the $a_{j}$ are determined by relations like (B.9) .

The next step is to compute the action on the $\varpi_{j}$ when going around the conifold $\psi=\left(\prod_{i=1}^{5} k_{i}^{k_{i} / d}\right)^{-1}$. (Due to the phase symmetry $\mathcal{A}$ the $d$ different singularities are identified and it is enough to study the above mentioned one.) Under transport around the singularity, $\varpi_{j}$ transforms according to [6]

$$
\varpi_{j}(\psi) \rightarrow \varpi_{j}(\psi)+c_{j} z^{0}(\psi)
$$

where $z^{0}=\varpi_{0}-\varpi_{1}$ vanishes at the singularity like $\left(\psi-\left(\prod_{i=1}^{5} k_{i}^{k_{i} / d}\right)^{-1}\right)$. Equivalently,

$$
\varpi_{j}=\frac{c_{j}}{2 \pi i} z^{0}(\psi) \log \left(\psi-\left(\prod_{i=1}^{5} k_{i}^{k_{i} / d}\right)^{-1}\right)+\ldots
$$


where the ellipses indicate terms analytic in a neighborhood of the conifold. Thus, the $c_{j}$ can be obtained by studying $\varpi_{j}$ for large $m$ in (B.7) along the lines of ref. [6] . We get

$$
\begin{aligned}
c_{j} & \stackrel{\text { def }}{=} \sum_{s=0}^{d-1} c_{j}(s) \\
& =\frac{1}{d} e^{\pi i\left(1-k_{1}\right)} \sum_{s=0}^{d-1} \alpha^{j s} \prod_{r=1}^{k_{1}-1}\left(\alpha^{s} e^{-2 \pi i r / k_{1}}-1\right) \prod_{i=2}^{5}\left(\alpha^{s k_{i}}-1\right) .
\end{aligned}
$$

Thus, (B.14) and (B.15) show that $\psi=\left(\prod_{i=1}^{5} k_{i}^{k_{i} / d}\right)^{-1}$ indeed is a conifold singularity. Eq. (B.15) can be simplified slightly by expanding $\prod_{r=1}^{k_{1}-1}\left(\alpha^{s} e^{-2 \pi i r / k_{1}}-1\right)$ giving us the final expression

$$
\begin{aligned}
c_{j} & =\frac{1}{d} \sum_{s=0}^{d-1} \alpha^{j s} \sum_{l=0}^{k_{1}-1} \alpha^{s l} \prod_{i=2}^{5}\left(\alpha^{s k_{i}}-1\right) \\
& =\sum_{l=0}^{k_{1}-1} \sum_{r=0}^{4} \sum_{i \in(2, \ldots, 5)_{r}}\left[\frac{k_{i}+l+j}{d}\right]^{r} .
\end{aligned}
$$

The last form is especially useful since it shows the integral structure and hence is preferable for actual computation, see examples in section 5 .

Note that for $k_{i}=1, i=1, \ldots, 5$ we recover the expression obtained in [6] . It is also worth pointing out that by further studying (B.16) one can show that $c_{0}=c_{1}=1$. Thus, for any given model we are now able write down both the monodromy matrix, $T$, associated to transport around $\psi=\left(\prod_{i=1}^{5} k_{i}^{k_{i} / d}\right)^{-1}$ using (B.13) and (B.16) as well as the phase symmetry matrix, $A$, see table B.1. Finally, the monodromy around $\psi=\infty$ is given by 6

$$
T_{\infty}=(A T)^{-1}
$$

$$
A=\left(\begin{array}{ccccc}
0 & 1 & 0 & \ldots & 0 \\
0 & 0 & 1 & \ldots & 0 \\
\vdots & \vdots & \vdots & \ddots & \vdots \\
0 & 0 & 0 & \ldots & 1 \\
a_{0} & a_{1} & a_{2} & \ldots & a_{q-1}
\end{array}\right) \quad T=\left(\begin{array}{cccccc|}
2 & -1 & 0 & 0 & \ldots & 0 \\
1 & 0 & 0 & 0 & \ldots & 0 \\
c_{2} & -c_{2} & 1 & 0 & \ldots & 0 \\
\vdots & \vdots & \vdots & \vdots & \ddots & \vdots \\
c_{q-1} & -c_{q-1} & 0 & 0 & \ldots & 1
\end{array}\right)
$$

Table B.1: Monodromy matrices $(A, T)$ associated to $\psi=0,\left(\prod_{i=1}^{5} k_{i}^{k_{i} / d}\right)^{-1}$. The number of linearly independent periods is $q$. 


\section{References}

[1] B.R. Greene and R. Plesser: "An Introduction to Mirror Manifolds", in Essays on Mirror Symmetry, and references therein, ed. S.-T. Yau (Intl. Press, Hong Kong, 1992).

[2] For a review and references, see L. Dixon: in Superstrings, Unified Theories and Cosmology 1987, eds. G. Furlan et al. (World Scientific, Singapore, 1988) p. 67-127.

[3] M. Kreuzer and H. Skarke: Commun. Math. Phys. 150 (1992) 137.

[4] A. Klemm and R. Schimmrigk : "Landau-Ginzburg String Vacua", CERN preprint CERN-TH 6459/92;

M. Kreuzer and H. Skarke: Nucl. Phys. B388 (1992) 113.

[5] M. Kreuzer and H. Skarke: "All Abelian Symmetries of Landau-Ginzburg Potentials", CERN-TH-6705/92.

[6] P. Candelas, X. de la Ossa, P. Green and L. Parkes: Nucl. Phys. B359 (1991) 21.

[7] D.R. Morrison: "Picard-Fuchs Equations and Mirror Maps For Hypersurfaces", in Essays on Mirror Symmetry, p.1, ed. S.-T. Yau (Intl. Press, Hong Kong, 1992);

A. Font: Nucl. Phys. B391 (1993)358;

A. Klemm and S. Theisen: "Mirror maps and instanton sums for complete intersections in weighted projective space", Munich University preprint LMU-TPW-93-08, Nucl. Phys. B389 (1993)153;

A. Libgober and J. Teitelbaum: "Lines on Calabi-Yau complete intersections, mirror symmetry and Picard-Fuchs equations" University of Illinois report (1992).

[8] P. Candelas, X. de la Ossa, A. Font, S. Katz, D.R. Morrison: "Mirror Symmetry for Two Parameter Models - I", CERN-TH. 6884/93, UTTG-15-93, NEIP-93-005, OSUM-93-1.

[9] S. Hosono, A. Klemm, S. Theisen and S.-T. Yau: "Mirror Symmetry, Mirror Map and Applications to Calabi-Yau Hypersurfaces", HUTMP-93/0801.

[10] T. Hübsch and S.-T. Yau: Mod. Phys. Lett. A7(1992)3277.

[11] V. Batyrev: "Dual polyhedra and mirror symmetry for Calabi-Yau hypersurfaces in toric varieties", Preprint, November 18, 1992.

[12] P.A. Griffiths: Ann. of Math. (2)90(1969).

[13] For a review see S. Ferrara: Mod. Phys. Lett. A6 (1991) 2175 and references therein.

[14] L. Dixon, V. Kaplunovsky and J. Louis: Nucl. Phys. B329 (1990) 27. 
[15] V. Batyrev and D. van Straten: "Generalized Hypergeometric Functions and Rational Curves on Calabi-Yau Complete Intersections in Toric Varieties", Preprint.

[16] P.S. Aspinwall, B.R. Greene and D.R. Morrison: Phys. Lett. B303 (1993) 249, "CalabiYau Moduli Space, Mirror Manifolds and Spacetime Topology Change in String Theory", Institute for Advanced Study report IASSNS-HEP-93/38.

[17] M. Dine, N. Seiberg, X.G. Wen and E. Witten: Nucl. Phys. B278 (1986)769, ibid. B289 (1987)319.

[18] P. Berglund and T. Hübsch: Nucl. Phys. B393 (1993)377, also in the Mirror Symmetry Workshop Proceedings, MSRI, Berkeley, May 1991.

[19] R. Bryant and P. Griffiths, Progress in Mathematics 36 p. 77, (Birkhäuser, Boston, 1983).

[20] P. Candelas, T. Hübsch and P. Green: Nucl. Phys. B330 (1990) 49.

[21] P. Candelas and X. de la Ossa: Nucl. Phys. B355 (1991)455.

[22] P.A. Griffiths: Bull. Amer. Math. Soc. 76 (1970) 228.

[23] A.C. Cadavid and S. Ferrara: Phys. Lett. B267 (1991)193.

[24] W. Lerche, D. J. Smit and N. P. Warner: Nucl. Phys. B372 (1992) 87.

[25] A. Ceresole, R. D'Auria, S. Ferrara, W. Lerche and J. Louis: Int.J.Mod.Phys. A8 (1993) 79 .

[26] P. Berglund, P. Candelas, X. de la Ossa, A. Font, T. Hübsch, D. Jančić and F. Quevedo, "Periods for Calabi-Yau and Landau-Ginzburg Vacua", CERN-TH. 6865/93, HUPAPP-93/3,NEIP 93-004, NSF-ITP-93-96, UTTG-13-93.

[27] P.S. Aspinwall, B.R. Greene and D.R. Morrison: "The Monomial-Divisor Mirror Map", IASSNS-HEP-93/43.

[28] W. Fulton: Introduction to toric varieties, Annals of Math. Studies, vol. 131, Princeton University Press, Princeton, 1993.

[29] P. Candelas, X. de la Ossa and S. Katz: "Mirror Symmetry for Calabi-Yau Hypersurfaces in Weighted $\mathbb{P}^{4}$ and an Extension of Landau-Ginzburg Theory", in preparation.

[30] V.V. Batyrev: Duke Math. Journ. 69 (1993) 349.

[31] P. Berglund and S. Katz: in preparation.

[32] T. Oda and H.S. Park: Tôhoku Math. J. (2) 43 (1991), 375.

[33] W. Lerche, C. Vafa and N. Warner: Nucl. Phys. B324 (1989) 427;

P. Candelas: Nucl. Phys. B298 (1988) 458. 
[34] C. Vafa : Mod. Phys. Lett. A6 (1991) 337.

[35] P. Green and T. Hübsch: Commun. Math. Phys. 113 (1987) 505.

[36] V. Batyrev: "Quantum Cohomology Rings of Toric Manifolds", Preprint.

[37] I. Dolgachev: "Weighted projective varieties", in Group Actions and Vector Fields, ed. J.B. Carrell, pages 34-71. Springer-Verlag, Berlin-Heidelberg-New York, 1982. Proceedings, Vancouver 1981. 